\title{
Outcome of patients with micropapillary urothelial carcinoma following radical cystectomy: ERBB2 (HER2) amplification identifies patients with poor outcome
}

\author{
Steven A Schneider ${ }^{1}$, William R Sukov ${ }^{2}$, Igor Frank ${ }^{1}$, Stephen A Boorjian ${ }^{1}$, \\ Brian A Costello ${ }^{3}$, Robert F Tarrell ${ }^{4}$, Prabin Thapa ${ }^{4}$, R Houston Thompson ${ }^{1}$, \\ Matthew K Tollefson ${ }^{1}$, R Jeffrey Karnes ${ }^{1}$ and John C Cheville ${ }^{2}$ \\ ${ }^{1}$ Department of Urology, Mayo Clinic, Rochester, MN, USA; ${ }^{2}$ Department of Laboratory Medicine and \\ Pathology, Mayo Clinic, Rochester, MN, USA; ${ }^{3}$ Internal Medicine (Urologic Oncology), Mayo Clinic, \\ Rochester, MN, USA and ${ }^{4}$ Health Sciences Research, Mayo Clinic, Rochester, MN, USA
}

\begin{abstract}
Micropapillary urothelial carcinoma exhibits amplification of the human epidermal growth factor receptor, ERBB2(HER2), and overexpression of the ERBB2 protein product. The clinical significance of this has yet to be established. The objective of this study was to examine ERBB2 amplification and protein expression in micropapillary urothelial carcinoma and stage-matched typical urothelial carcinoma treated by radical cystectomy to assess the frequency of amplification and protein expression, and to determine the association with cancer-specific survival. Pathologic material and data from patients undergoing cystectomy at Mayo Clinic between 1980 and 2008 were reviewed. ERBB2 amplification by fluorescence in situ hybridization (FISH) and protein expression by immunohistochemistry were assessed. Univariate and multivariate Cox proportional hazards regression models were used to evaluate for associations of ERBB2 amplification and protein expression with survival. ERBB2 amplification was identified in $9(15 \%)$ of 61 micropapillary carcinomas compared with $9(9 \%)$ of 100 urothelial carcinomas. In patients with micropapillary carcinoma, ERBB2 amplification was associated with a nearly threefold increased risk of cancer death. ERBB2 amplification (hazard ratio 4.3; $\boldsymbol{P}=0.0008$ ) remained associated with an increased risk of death from bladder cancer among patients with micropapillary urothelial carcinoma on multivariate analysis. The association of cancer-specific survival and ERBB2 amplification was not seen in patients with urothelial carcinoma. ERBB2 immunohistochemistry correlated with ERBB2 amplification but there was no association of ERBB2 protein expression and survival. ERBB2 amplification is more frequent in micropapillary urothelial carcinoma than typical urothelial carcinoma, and patients with micropapillary carcinoma who have ERBB2 amplification have worse cancer-specific survival than those who do not. Identification of ERBB2 amplification in micropapillary carcinoma could provide important prognostic information and possibly provide a role for ERBB2 targeted therapy.
\end{abstract}

Modern Pathology (2014) 27, 758-764; doi:10.1038/modpathol.2013.201; published online 1 November 2013

Keywords: bladder cancer; cystectomy; ERBB2; ERBB2; micropapillary; urothelial carcinoma

Micropapillary urothelial carcinoma is a unique variant of urothelial carcinoma that comprises $<6 \%$ of all bladder cancers. ${ }^{1-3}$ Several studies demonstrated that micropapillary urothelial carcinoma is

Correspondence: Dr JC Cheville, MD, Department of Laboratory Medicine and Pathology, Mayo Clinic, 200 First Street, SE, Rochester, MN 55905, USA.

E-mail: cheville.john@mayo.edu

Received 20 August 2013; revised 10 September 2013; accepted 15 September 2013; published online 1 November 2013 diagnosed at a more advanced stage, and is associated with adverse outcome relative to typical urothelial carcinoma. ${ }^{4-8}$ However, a recent study found that, although patients with micropapillary urothelial carcinoma presented at higher stage than patients with typical urothelial carcinoma, the outcomes following radical cystectomy were no different when matched for stage and other clinicopathologic variables. ${ }^{9}$

In addition to unique histologic features, studies have shown that micropapillary urothelial carcinoma 
exhibits amplification of the human epidermal growth factor receptor (ERBB2 or HER2) in over $40 \%$ of cases. ${ }^{10}$ ERBB2 is a type-I transmembrane growth factor receptor that activates intracellular signaling pathways in response to extracellular signals. It was first implicated in the pathogenesis of human breast cancer 25 years ago. ${ }^{11}$ In patients with breast cancer, ERBB2 amplification is associated with poor prognosis. ${ }^{12}$ However, ERBB2-targeted therapy with the anti-ERBB2 antibody trastuzumab has resulted in significantly longer survival when combined with standard chemotherapy in patients whose breast tumors exhibit ERBB2 amplification compared with patients whose tumor lack ERBB2 amplification. $^{12,13}$ ERBB2 amplification has been identified in other tumor types, although its role in treatment for these malignancies is yet to be defined. ${ }^{10,14-16}$

Although ERBB2 amplification has been identified in micropapillary urothelial carcinoma, its frequency and association with outcome has not been established in patients undergoing curative surgery. The objective of this study was to examine ERBB2 amplification and protein expression in a consecutive series of patients with micropapillary urothelial carcinoma undergoing radical cystectomy to determine the frequency of amplification and protein expression, and their association with outcome. In addition, ERBB2 and protein status was compared between patients with micropapillary urothelial carcinoma and patients with typical urothelial carcinoma matched for the prognostic clinical and pathologic variables.

\section{Materials and methods}

After Institutional Review Board approval was obtained, we reviewed the Mayo Clinic Cystectomy Registry of patients who underwent radical cystectomy at our institution between 1980 and 2008 . Radical cystectomy was performed by various surgeons using standard techniques. Tissue slides and blocks are retained permanently on all patients, and all cystectomy specimens from 1980 to 2008 were re-reviewed by a urologic pathologist, and 61 cases of micropapillary urothelial carcinoma were identified. Inclusion of cases into the study required any micropapillary component in a urothelial carcinoma. These 61 cases were matched for stage, age of patient, ECOG status, receipt of perioperative chemotherapy and decade of surgery, with 100 cases of typical urothelial carcinoma. All cases were re-reviewed a second time by two pathologists (WRS and JCC) independently and blinded to the prior diagnoses to ensure the appropriate diagnosis of micropapillary carcinoma. Consensus agreement was reached on all cases in this study. Staging followed the 2010 American Joint Committee on Cancer/Union Internationale Contre le Cancer 7th edition TNM classification. ${ }^{17}$ As all these specimens were obtained before the 2010 staging system, retrospective staging was performed as described previously. ${ }^{18}$ Briefly, pT1 and pT2 classification was obtained by microscopic slide review as staging parameters are based on microscopic features. In the case of pT3 tumors, the tissue slides (where gross features of the bladder and tumor are retained) were examined macroscopically, and the presence or absence of a gross lesion was determined, and findings confirmed microscopically.

The retrospective nature of this study precluded a standardized postoperative follow-up protocol in all patients. However, follow-up after radical cystectomy at our institution has generally been recommended quarterly for the first 2 years after surgery, semiannually for the next 2 years, and annually thereafter in patients without evidence of disease recurrence. Vital status was identified from death certificates or physician correspondence. For patients followed elsewhere, the Mayo Clinic Cystectomy Registry monitors outcomes annually by correspondence to the patient and treating physician.

Clinicopathologic demographics for patients with micropapillary cancer were reported and compared with a group of patients with typical urothelial carcinoma on the basis of age, gender, T-stage, N-stage, ECOG performance status, receipt of neoadjuvant chemotherapy, postoperative chemotherapy and radiation and surgical margin status.

\section{ERBB2 Testing Methods and Interpretive Criteria}

ERBB2 (HER2) by fluorescence in situ hybridization (FISH). ERBB2 tests approved by the US Food and Drug Administration (FDA) were used for FISH. ERBB2 amplification was assessed in formalinfixed, paraffin-embedded $5-\mu \mathrm{m}$ sections using the PathVysion HER-2 DNA Probe Kit (HER2 and centromere 17 probes; Abbott Molecular, Des Plaines, IL, USA), as described. Whole tumor sections from cystectomy specimens were used, and the complete section was scanned by certified cytogenetic technologists to detect any subpopulation of amplified cells. A total of 60 representative nuclei from the invasive tumor were scored, with an overall evaluation performed by an cytogeneticist. ${ }^{19,20}$ A specimen with an ERBB2/centromere 17 ratio $\geq 2.0$ in invasive cells was classified as ERBB2 amplified in accordance with the criteria developed for classification of ERBB2 and centromere 17 abnormalities as described previously. ${ }^{19,21}$

Chromosome 17 gains and losses were determined using centromere 17 signal patterns based on methodology and cutoffs that we previously validated using two large independent breast cancer sets. Accordingly, polysomy 17 (gain) was defined as three centromere 17 signals in more than $30 \%$ of nuclei; monosomy 17 (loss) was defined as one centromere 17 signal in more than $60 \%$ of nuclei; and all other cases were considered normal. ${ }^{20}$ Both 
Table 1 Clinicopathologic features of patients with micropapillary urothelial carcinoma and typical urothelial carcinoma

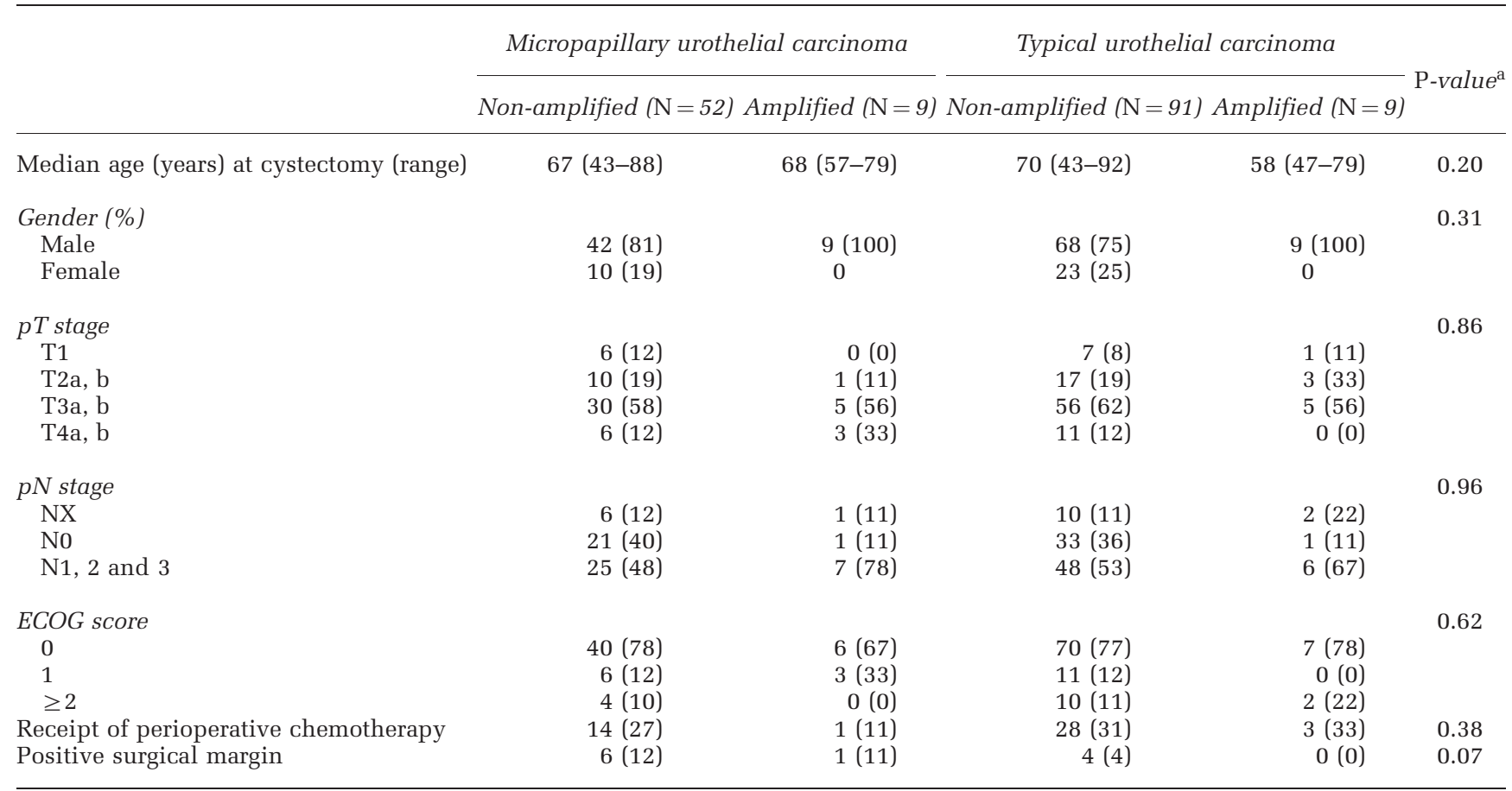

${ }^{\mathrm{a}} P$-values reflect comparison of patients with MP UC and UC.

cutoffs clearly distinguish chromosome 17 polysomic and monosomic cancers from cancers without chromosome 17 centromere anomalies. ${ }^{22}$ All categorization thresholds were selected to reduce the rate of false-positive findings for gene amplification, gene deletion and chromosome loss or gain. Quality control of the ERBB2 FISH test is routinely assessed according to standard CAP and American College of Medical Genetics guidelines. ${ }^{20,23-25}$

\section{ERBB2 Heterogeneity by FISH}

Following CAP breast cancer guidelines, ${ }^{20}$ a sample was considered to have heterogeneous ERBB2 amplification if there were $>5 \%$ but $<50 \%$ infiltrating tumor cells with an ERBB2/centromere 17 ratio $>2.2$ (results were the same for a 2.0 cutpoint). An ERBB2/centromere ratio was determined separately for the amplified and non-amplified subpopulation of each heterogeneous tumor. The primary ERBB2/centromere 17 ratio for each heterogeneous case was determined by calculating a mean ratio across the amplified and non-amplified subpopulations weighted by the percentage that each subpopulation comprised the entire tumor. $E R B B 2$ heterogeneous tumors were further characterized by the distribution of the amplified subpopulation-that is, clustered (amplified cells adjacent to each other, tending to be arranged in groups) versus diffuse (amplified cells scattered among the non-amplified cells and relatively evenly distributed throughout the tumor).
ERBB2 immunohistochemistry was performed using the HercepTest (Dako) according to the manufacturer's recommendations. Immunohistochemistry staining was scored according to recommendations of the American Society of Clinical Oncology/College of American Pathology guidelines. ${ }^{24}$

\section{Statistical Analysis}

The Wilcoxon's rank-sum and $\chi^{2}$ tests were used to compare variables between groups. For survival analysis, the outcome variable was cancer-specific survival, defined as the time from surgery to death related to index cancer and was censored at the date of death as a result of postoperative complications or other non-malignant causes. Cancer-specific survival was estimated using the Kaplan-Meier method and compared with the log-rank test. Associations of features with time to death from bladder cancer was evaluated using Cox proportional hazards regression models, and summarized with hazard ratios (HR) and 95\% confidence intervals (CI). All $P$-values are two-sided, with a $P<0.05$ considered statistically significant. Analyses were conducted in SAS version 9.1 (SAS Institute, Cary, NC, USA).

\section{Results}

The clinicopathologic features of the patients with micropapillary carcinoma and typical urothelial carcinoma are listed in Table 1. There were 61 
Table 2 Univariate associations with cancer-specific survival among patients with micropapillary urothelial carcinoma

\begin{tabular}{|c|c|c|c|}
\hline Parameter & $\begin{array}{l}\text { Hazard } \\
\text { ratio }\end{array}$ & $\begin{array}{c}95 \% \text { Confidence } \\
\text { interval }\end{array}$ & P-value \\
\hline Age at surgery & 1.0 & $0.98-1.04$ & 0.7 \\
\hline $\begin{array}{l}\text { pT stage (reference } \\
\leq \mathrm{T} 1)\end{array}$ & 1.6 & $0.8-3.3$ & 0.16 \\
\hline $\begin{array}{l}\text { pN stage (reference } \\
\text { pNo) }\end{array}$ & 2.2 & $1.1-4.6$ & 0.03 \\
\hline ECOG & 2.6 & $1.9-3.1$ & 0.002 \\
\hline $\begin{array}{l}\text { Perioperative } \\
\text { chemotherapy }\end{array}$ & 1.1 & $0.6-2.4$ & 0.63 \\
\hline ERBB2 amplification & 4.2 & $1.9-9.7$ & $<0.001$ \\
\hline
\end{tabular}

patients within the micropapillary (Table 2) cancer and 100 with urothelial carcinoma. Median followup of all patients was 12.8 years. The micropapillary urothelial carcinoma and urothelial carcinoma groups were matched such that there were no statistical differences between the cohorts with regard to age, gender, ECOG performance status, length of follow-up, stage or reception of perioperative (neoadjuvant) chemotherapy. With regard to postoperative treatment, $14(23 \%)$ patients with micropapillary carcinoma received chemotherapy compared with $41(25 \%)$ of patients with typical urothelial carcinoma, and postoperative radiation was given to $1(1.6 \%)$ with micropapillary cancer and $2(1.2 \%)$ patients with typical urothelial carcinoma. In the cases of micropapillary urothelial carcinoma, the majority of cases (55 of $73 ; 75 \%$ ) were composed entirely of micropapillary carcinoma, and the range of the micropapillary carcinoma component in the remaining cases ranged from 5 to $90 \%$. The other component in these cases was typical urothelial carcinoma.

ERRB2 amplification was identified in $9(15 \%)$ of 61 micropapillary urothelial carcinoma compared with $9(9 \%)$ of 100 typical urothelial carcinomas (Figure 1). No heterogeneity of ERBB2 amplification was identified. Patients with ERBB2-amplified micropapillary carcinomas were more likely to have positive lymph nodes at the time of cystectomy than patients with non-amplified micropapillary carcinomas, $78 \%$ versus $48 \%$, respectively, but this did not reach statistical significance $(P=0.078)$. There were no differences in patient, age, gender, pT stage, ECOG status or margin status between patients with amplified and non-amplified tumors. In addition, there were no differences in the morphologic features (nuclear or architectural features) of tumors with and without amplification. The 5-year cancerspecific survival of patients with micropapillary urothelial carcinoma with ERBB2 amplification was $0 \%$, as opposed to patients with micropapillary urothelial carcinoma without amplification whose 5 -year cancer-specific survival was $40 \% \quad(P<0.001)$ (Figure 2). There was no statistically significant difference in cancer-specific survival between

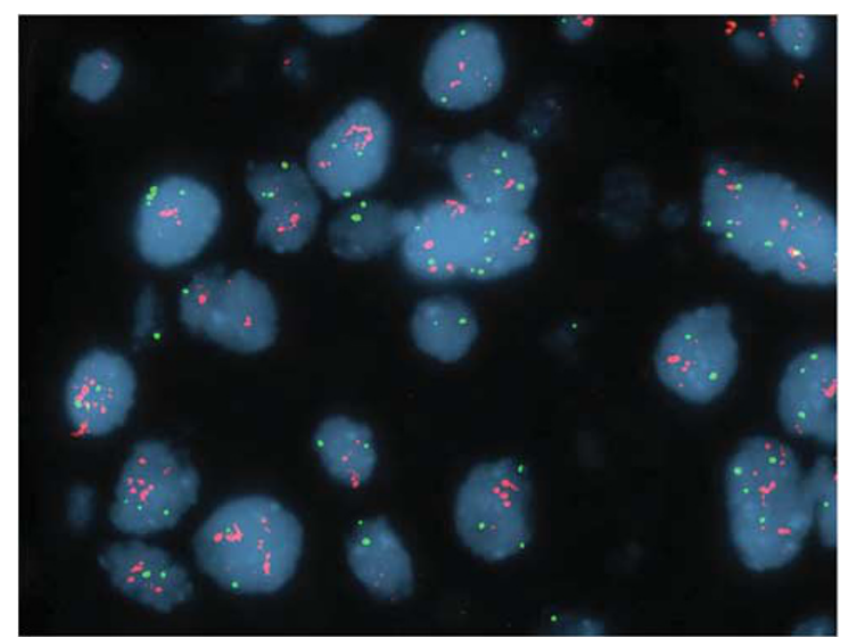

Figure 1 A case of micropapillary urothelial carcinoma with ERBB2 amplification. Red probe indicates copies of ERBB2; green probe indicates chromosome 17 centromere.

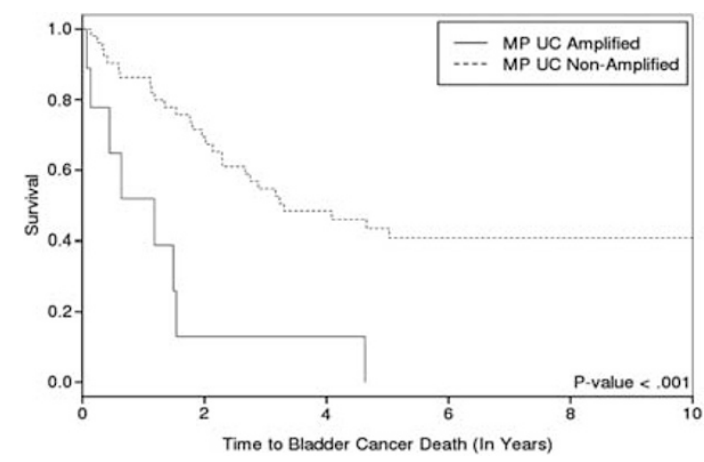

Figure 2 Cancer-specific survival of patients with ERBB2-amplified micropapillary urothelial carcinoma (MP UC) compared to patients with -non-amplified micropapillary carcinoma.

patients with typical urothelial carcinoma with amplification and without amplification (Figure 3). Associations of clinical and pathologic features and ERBB2 amplification with cancer-specific survival are summarized in Tables 3 and 4 . On univariate analysis, ERBB2 amplification was associated with a nearly threefold increased risk of death from bladder cancer. On multivariate analysis, ERBB2 amplification (HR 4.3; $P=0.0008$ ) and ECOG score (HR 2.2; $P=0.007)$ remained significantly associated with death from bladder cancer.

Immunohistochemistry results are provided in Table 4 and illustrated in Figure 4. In patients with micropapillary carcinoma and typical urothelial carcinoma, there was no significant association of immunohistochemistry scores with cancer-specific survivals when comparing intensity staining scores 0,1 versus 2,3 as typically done in breast cancer. There was a strong association of ERBB2 amplification and ERBB2 immunohistochemical scores 2 and 3 . Fifteen $(83 \%)$ of 18 bladder cancers that showed ERBB2 amplification had ERBB2 immunohistochemical scores of 2 and 3 . Three cases of 


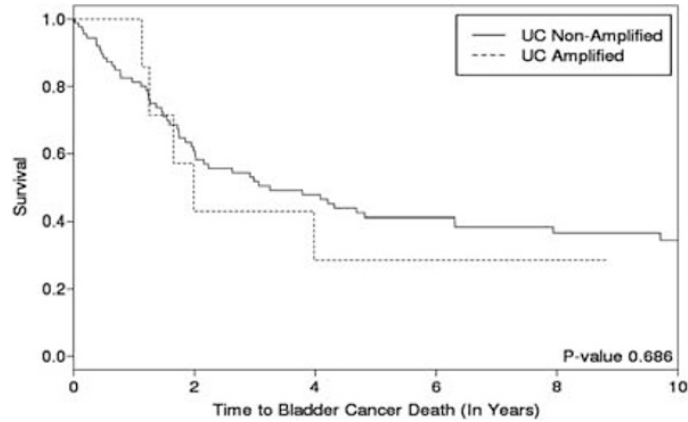

Figure 3 Cancer-specific survival of patients with ERBB2-amplified typical urothelial carcinoma (UC) compared to patients with non-amplified typical urothelial carcinoma.

Table 3 Multivariate associations with cancer-specific survival in patients with micropapillary urothelial carcinoma

\begin{tabular}{lccc}
\hline Parameter & $\begin{array}{c}\text { Hazard } \\
\text { ratio }\end{array}$ & $\begin{array}{c}\text { 95\% Confidence } \\
\text { interval }\end{array}$ & P-value \\
\hline $\begin{array}{l}\text { ERBB2 } \\
\text { amplification }\end{array}$ & 4.3 & $1.8-10.2$ & 0.0008 \\
ECOG & 2.2 & $1.4-3.9$ & 0.0007 \\
\hline
\end{tabular}

Table 4 Immunohistochemical results in micropapillary urothelial carcinoma and typical urothelial carcinoma and comparison to ERBB2 amplification

\begin{tabular}{lccc}
\hline Micropapillary \\
urothelial \\
$\begin{array}{l}\text { Immunohistochemical } \\
\text { scorcinoma no. } \\
(\%), \mathrm{N}=61\end{array}$ & $\begin{array}{c}\text { Typical } \\
\text { carcinomal } \\
\text { no. }(\%), \\
\mathrm{N}=100\end{array}$ & $\begin{array}{c}\text { No. of } \\
\text { patients with } \\
\text { ERBB2 } \\
\text { amplification } \\
(\%), \mathrm{N}=18\end{array}$ \\
\hline 0 & $9(15)$ & $54(54)$ & $2(11)$ \\
1 & $21(35)$ & $22(22)$ & $1(5)$ \\
2 & $15(25)$ & $14(14)$ & $3(17)$ \\
3 & $15(25)$ & $10(10)$ & $12(67)$ \\
\hline
\end{tabular}

ERBB2-amplified cancer had a score $<2$. However, immunohistochemical scores 2 and 3 were present in $39(27 \%)$ of 143 patients with micropapillary carcinoma and urothelial carcinoma that did not have ERBB2 amplification.

\section{Discussion}

In this study involving radical cystectomy patients, ERBB2 amplification was significantly associated with diminished cancer-specific survival among patients with micropapillary urothelial carcinoma. To our knowledge, this represents the first time that ERBB2 amplification has been observed to predict for outcome among patients with the rare variant of urothelial carcinoma. Furthermore, these results suggest that therapeutic manipulation of ERBB2 may hold promise for patients harboring the aggressive micropapillary urothelial carcinoma.

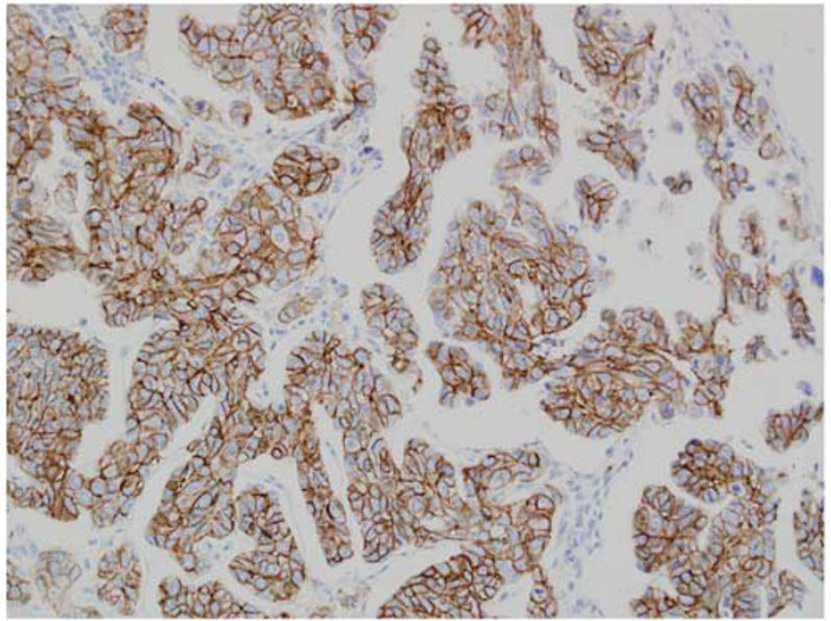

Figure 4 Micropapillary urothelial carcinoma with ERBB2 score of 3 .

Micropapillary urothelial carcinoma is an unusual variant of bladder cancer that comprises 0.7 to $6 \%$ of all bladder cancers. It was initially described in 1994 and named for its resemblance to papillary serous carcinoma of the ovary. ${ }^{3}$ Several studies have suggested that micropapillary urothelial carcinoma has a particularly aggressive course compared with typical urothelial carcinoma, but these studies have been limited by small patient numbers or nonstandardized treatment and follow-up. Recently, we examined our experience of micropapillary urothelial carcinoma treated by radical cystectomy and showed that micropapillary urothelial carcinoma was associated with advanced locoregional spread at the time of surgery when compared to patients with typical urothelial carcinoma. ${ }^{9}$ However, when matched for stage and other prognostic clinicopathologic features, patients with micropapillary carcinoma had the same outcome as patients with typical urothelial carcinoma. In this study, we further define the behavior of micropapillary urothelial carcinoma and show that ERBB2 amplification identifies a group of patients with particularly adverse outcome.

Although this study requires external validation, our findings have several important clinical implications. For one, the assessment of ERBB2 status could provide important prognostic information for patients under consideration for surgery. In a tumor that has been assumed to be particularly aggressive, the absence of ERBB2 amplification suggests an outcome following cystectomy similar to patients with similar stage typical urothelial carcinoma. The presence of ERBB2 amplification also suggests a higher likelihood of lymph node involvement that may alter the surgical approach and a greater probability of disease progression that would affect perioperative treatment and follow-up. There also implications with regard to targeted therapy. The role of trastuzumab in the treatment of breast cancer is well established. Recently, a survival benefit has 
been reported in advanced $E R B B 2$-amplified gastric cancer treated by anti-EBBR2 monoclonal antibody. ${ }^{26}$ There is potential that patients with ERBB2-amplified bladder cancers may benefit from trastuzumab therapy, and that the frequency of amplification can be expected to be higher in micropapillary carcinoma than typical urothelial carcinoma. It is important to note that, although relatively low, we detected a frequency of ERBB2 amplification of approximately $9 \%$ in typical urothelial cancers similarly to other studies. ${ }^{16}$ Although the number of patients with typical urothelial carcinoma and ERBB2 amplification is small, we did not identify a worse outcome in those patients when compared with patients who had non-amplified tumors. Clinical trials are needed to determine the effectiveness of anti-ERBB2 therapy in these patients, and trials would benefit from central pathology review to stratify patients by bladder cancer subtype and trials will require standardized assessment of ERBB2 tumor status.

As expected, immunohistochemistry for ERBB2 had a relatively high positive predictive value for the identification of bladder cancers that show ERBB2 amplification with 15 of 18 (83\%) ERBB2amplified tumors showing high (score 2 and 3 ) ERBB2 overexpression. However, a large proportion of tumors showing score 2 and 3 overexpression did not show ERBB2 amplification (39 of 142 nonamplified cases). The significance of ERBB2 overexpression in non-amplified tumors is not known. It will be important in future prospective clinical studies to explore whether there is any potential benefit from trastuzumab in patients not only with ERBB2-amplified tumors but also patients with ERBB2-non-amplified tumors that show ERBB2 overexpression. It is of note that we did not identify an association of cancer-specific survival and ERBB2 protein expression in patients with micropapillary or typical urothelial carcinoma.

We report a lower frequency of ERBB2 amplification in micropapillary carcinoma than other groups. In particular, Ching et $a l^{10}$ reported that $8(42 \%)$ of 19 micropapillary carcinomas showed amplification and $13(68 \%)$ exhibited score 2 and 3 by immunohistochemistry compared with $15 \%$ and $50 \%$, respectively, in our series. In addition, Ching et al found no association of ERBB2 amplification with outcome. There are several potential explanations for these differences. The study by Ching et al evaluated only 20 patients, four that received transurethral resection as the only surgical intervention, whereas 16 underwent radical cystectomy. Fourteen $(88 \%)$ of the 16 patients had regional lymph node involvement at surgery and the remaining two were suspected of having involvement based on radiology, and 12 of their patients died over follow-up of 14 months. In our series, $32(52 \%)$ patients with micropapillary carcinoma had regional lymph node involvement, and patients with lymph node involvement had a higher frequency of ERBB2 amplification. Of the 9 patients with amplified ERBB2 tumors, 7 (78\%) had regional lymph node involvement compared with $25(48 \%)$ of 52 patients with non-amplified tumors. These findings suggest that the study by Ching et al consisted of patients with more advanced disease, and as a consequence their series showed a higher frequency of ERBB2 amplification. There were also differences in methodology between the two studies. Ching et al used a modified chromagenic in situ hybridization technique applied to tissue microarrays in contrast to the FDA-approved FISH technique performed on whole tumor sections in our analysis. Finally, Ching et al performed immunohistochemistry with a primary antibody from Ventana while we utilized the FDA-approved Herceptest from Dako. It is difficult to assess the impact of these different methodologies on results but these differences emphasize the need for a standardized approach to determine ERBB2 amplification across clinical trials.

There are several limitations to this study. Although the number of patients undergoing radical cystectomy for micropapillary urothelial carcinoma is relatively large compared with other studies, it remains quite limited. In addition, the study was retrospective and non-randomized. Our findings will, therefore, require validation either through a large multi-institutional retrospective study or preferably through a prospective clinical trial examining the prognostic significance of ERBB2 amplification and the application of targeted therapy in the setting of cystectomy.

\section{Acknowledgments}

This project was supported in part by funding from the Mayo Clinic Center for Individualized Medicine.

\section{Disclosure/conflict of interest}

The authors declare no conflict of interest.

\section{References}

1 Johansson SL, Borghede G, Holmang S. Micropapillary bladder carcinoma: a clinicopathological study of 20 cases. J Urol 1999;161:1798-1802.

2 Alvarado-Cabrero I, Sierra-Santiesteban FI, MantillaMorales A, et al. Micropapillary carcinoma of the urothelial tract. A clinicopathologic study of 38 cases. Ann Diagn Pathol 2005;9:1-5.

3 Amin MB, Ro JY, el-Sharkawy T, et al. Micropapillary variant of transitional cell carcinoma of the urinary bladder. Histologic pattern resembling ovarian papillary serous carcinoma. Am J Surg Pathol 1994;18: 1224-1232.

4 Ghoneim IA, Miacinovic R, Stephenson AJ, et al. Neoadjuvant systemic therapy or early cystectomy? Single-center analysis of outcomes after therapy for 
patients with clinically localized micropapillary carcinoma of the bladder. Urology 2011;77:867-870.

5 Comperat E, Roupert M, Yaxley J, et al. Micropapillary urothelial carcinoma of the urinary bladder: a clinicopathological analysis of 72 cases. Pathology 2010;42: 650-654.

6 Kamat Am, Dinney CP, Gee JR, et al. Micropapillary bladder cancer: a review of the University of Texas M.D. Anderson Cancer Center experience with 100 consecutive patients. Cancer 2007;110:62-67.

7 Edgerton N, Sirintrapun SJ, Munoz M, et al. Micropapillary urothelial carcinoma of the urinary bladder: a clinicopathological analysis of 24 cases. Int J Urol 2011;18:49-54.

8 Lopez-Beltran A, Montironi R, Blanca A, et al. Invasive micropapillary urothelial carcinoma of the bladder. Hum Pathol 2010;41:1159-1164.

9 Wang JK, Boorjian SA, Cheville JC, et al. Outcomes following radical cystectomy for micropapillary bladder cancer versus pure urothelrial carcinoma: a matched cohort analysis. World J Urol 2012;30:801-806.

10 Ching CB, Amin MB, Tubbs RR, et al. HER2 gene amplification occurs frequently in micropapillary variant of urothelial carcinoma: analysis by dualcolor in situ hybridization. Mod Pathol 2011;24: 1111-1119.

11 Moasser M. The oncogene HER2: its signaling and transforming functions and its role in human cancer pathogenesis. Oncogene 2007;26:6469-6487.

12 Slamon DJ, Clark GM, Wong SG, et al. Human breast cancer: correlation of relapse and survival with amplification of the HER2/neu oncogene. Science 1987;235:177-182.

13 Slamon DJ, Leyland-Jones B, Shak S, et al. Use of chemotherapy plus a monoclonal antibody against HER2 for metastatic breast cancer that overexpresses HER2. N Engl J Med 2001;344:783-792.

14 Tolmachev V. Imaging of Her-2 overexpression in tumors for guiding therapy. Curr Pharm Des 2008;14: 2999-3019.

15 Yoon H, Shi Q, Sukov W, et al. Adverse prognostic impact of intratumor heterogeneous HER2 gene amplification in patients with esophageal adenocarcinoma. J Clin Oncol 2012;30:3932-3938.

16 Lae M, Couturier J, Oudard S, et al. Asessing HER2 gene amplification as a potential target for therapy in invasive urothelial bladder cancer with a standardized methodology: results in 1005 patients. Ann Oncol 2010;21:815-819.

17 AJCC AJCC Cancer Staging Manual, 7th edn. Springer: Chicago, IL, USA, 2010.

18 Zarei S, Frank I, Boorjian SA, et al. Prognostic significance of measured depth of invasion or urothelial carcinoma of the bladder compared to the 2010 American Joint Committee on Cancer pT2 and pT3 classification. J Urol 2012;188:1706-1711.

19 Perez EA, Suman VJ, Davidson NE, et al. HER2 testing by local central, and reference laboratories in specimens from the North Central Cancer Treatment Group N9831 intergroup adjuvant trial. J Clin Oncol 2006; 24:3032-3038.

20 Wolf AC, Hammond ME, Schwartz JN, et al. American Society of Clinical Oncology/College of American Pathologists guideline reommendations for human epidermal growth factor receptor 2 testing in breast cancer. J Clin Oncol 2007;25:118-145.

21 Vance GH, Barry TS, Bloom KJ, et al. Genetic heterogenecity in HER2 testing in breast cancer: panel summary and guidelines. Arch Pathol Lab Med 2009; 133:611-612.

22 Perez EA, Reinholz MM, Hillman DW, et al. HER2 and chromosome 17 effect on patient outcome in the N9831 adjuvant trastuzumab trial. J Clin Oncol 2010;28: 4307-4315.

23 Mascarello JT, Brothman AR, Davison K, et al. Proficiency testing for laboratories performing flourescence in situ hybridization with chromosome specific DNA probes. Arch Pathol Lab Med 2002;126:1458-1462.

24 Cell Markers And Cytogenetics Committees College Of American Pathologists. Cell Markers and Cytogenetics Committees of the College of American Pathologists: Clinical laboratory assays for HER-2/neu amplification and overexpression: Quality assurance, standardization and proficiency testing. Arch Pathol Lab Med 2002;126:803-808.

25 Westgard JO, Barry PL, Hunt MR, et al. A multi-rule Shewhart chart for quality control in clinical chemistry. Clin Chem 1981;27:493-501.

26 Bang YJ, Van Cutsem E, Feyereisiova A, et al. Trastuzumab in combination with chemotherapy versus chemotherapy alone for treatment of HER2-positive gastric or gastro-oesophageal junction cancer (ToGA): a phase 3, open label, randomized controlled trial. Lancet 2010;18:53-59. 